\title{
ASPECTOS PSICOSSOCIAIS DE ACADÊMICOS DE ENFERMAGEM DURANTE A PANDEMIA DA COVID-19
}

\author{
Drielly da Silva Galvão ${ }^{1}$ \\ Alaidistania Aparecida Ferreira ${ }^{1}$ \\ Nailu Flor Chenini de Carvalho Reis ${ }^{1}$ \\ Silvani Vieira Cardoso ${ }^{1}$ \\ Thiago do Nascimento Reis ${ }^{1}$
}

\author{
https://orcid.org/0000-0002-1329-0612 \\ https://orcid.org/0000-0001-8553-7269 \\ https://orcid.org/0000-0001-7888-596X \\ https://orcid.org/0000-0002-4678-0220 \\ https://orcid.org/0000-0002-7437-6305
}

Objetivo: Relatar a experiência do isolamento social de acadêmicos de enfermagem no período de pandemia de COVID-19. Método: Trata-se de um relato de experiência realizado mediante adesão de novas estratégias para elaboração de estudos, em razão do atual cenário vivido, as discussões do relato se deram por videoconferências em plataformas virtuais que permitiram o debate crítico do tema de acordo com as medidas de isolamento social. Principais resultados: Identificou-se agentes estressores advindos do isolamento social que comprometeram a saúde mental e física dos acadêmicos do estudo. Considerações finais: Para os acadêmicos, além dos sentimentos prejudiciais, sucedeu a expectativa de um futuro melhor, com pensamentos empáticos para com a sociedade e convicção que a Enfermagem será mais valorizada, bem como os profissionais que atuam nela.

Descritores: Estudantes de Enfermagem; Isolamento Social; Pandemias.

\section{PSYCHOSOCIAL ASPECTS OF NURSING STUDENTS DURING THE COVID-19 PANDEMIC}

Objective: To report the experience of social isolation of nursing students in the pandemic period of COVID-19. Method: This is an experience report carried out through the adherence of new strategies for the elaboration of studies, due to the current scenario, the discussions of the report took place by videoconferences on virtual platforms that allowed the critical debate of the theme according to the social isolation measures. Main results: Stressors from social isolation were identified that compromised the mental and physical health of the students in the study. Final considerations: For academics, in addition to harmful feelings, there was an expectation of a better future, with empathetic thoughts towards society and the belief that Nursing will be more valued, as well as the professionals who work in it.

Descriptors: Nursing Students; Social Isolation; Pandemics.

\section{ASPECTOS PSICOSOCIALES DE LOS ESTUDIANTES DE ENFERMERİA DURANTE LA PANDEMIA DEL COVID-19}

Objetivo: Informar la experiencia de aislamiento social de estudiantes de enfermería en el período pandémico de COVID-19. Metodo: Este es un informe de experiencia llevado a cabo mediante la adhesión de nuevas estrategias para la elaboración de estudios, debido al escenario actual, las discusiones del informe se llevaron a cabo por videoconferencias en plataformas virtuales que permitieron el debate crítico del tema de acuerdo con el medidas de aislamiento social. Principales resultados: Se identificaron factores estresantes del aislamiento social que comprometieron la salud mental y física de los estudiantes en el estudio. Consideraciones finales: Para los académicos, además de los sentimientos nocivos, había una expectativa de un futuro mejor, con pensamientos empáticos hacia la sociedad y la creencia de que la Enfermería será más valorada, así como los profesionales que trabajan en ella.

Descriptores: Estudiantes de Enfermería; Aislamiento Social; Pandemias.

${ }^{1}$ Universidade Federal do Amazonas - UFAM, Manaus, AM.

Autor Correspondente: Drielly da Silva Galvão Email: Driellygalvao07@hotmail.com

Recebido: 10/6/2020 Aceito: 17/7/2020 


\section{INTRODUÇÃO:}

A COVID-19 vírus (Sars-CoV-2) é uma doença que apresenta um quadro clínico que varia de infecções assintomáticas a quadros respiratórios graves causadas pelo coronavírus SARS-CoV-2 descoberto no final do ano de 2019 na cidade Wuhan, na China ${ }^{1}$.

Com o surgimento e identificação do vírus Sars-CoV-2, agente etiológico da COVID-19 e sua disseminação em vários países, e por ser considerado um vírus respiratório altamente contagioso, algumas medidas de saúde visando a diminuição do contágio foram adotadas, como isolamento social, distanciamento social e a quarentena para a população. Essas medidas consistem em um distanciamento de pessoas a fim de evitar contato, e assim, diminuir o risco de contaminação pelo vírus, o que interfere diretamente ou indiretamente na questão emocional ${ }^{2}$.

O COVID-19 foi diagnosticado no Brasil, no dia 26 de fevereiro de 2020, na cidade de São Paulol. Em Manaus, o primeiro paciente confirmado foi no dia 13 de março de 2020, em um bairro da região sul da cidade. Acredita-se que nesse período, o vírus não possuía potencial de disseminação em uma região com altas temperaturas como Manaus, porém, ele adaptou-se e após 15 dias do primeiro caso, já contabilizávamos mais de 80 casos confirmado ${ }^{3-4}$.

Com esse acometimento da saúde pública e privada, adotou-se, em Manaus, o isolamento domiciliar, a fim de prevenir a dispersão do vírus, assim sendo, muitos serviços foram suspensos por tempo indeterminado, como fechamento de espaços públicos e de entretenimento, escolas, universidades, academias, comércios e algumas empresas. A rotina, de milhares de pessoas sofreram alterações, não planejadas, ao ficarem reclusas em suas residências. Os estudantes de universidades públicas, que tinham atividades diárias e em sua maioria em tempo integral, tiveram suas aulas suspensas por tempo indeterminado. A mudança de hábitos sociais são fatores que contribuem, desencadeiam e agravam o estresse e a ansiedade, a ociosidade então surgiu, e com ela sinais e sintomas de estresse.

O estresse pode manifestar-se de diversas formas, a ansiedade e a ociosidade surgem a partir de um ou mais fatores estressores. A mudança nos hábitos, de rotina, as preocupações, geram a ansiedade. O tempo e a duração da exposição ao estresse é que irá determinar o grau de comprometimento da saúde ${ }^{5}$. Durante a exposição prolongada ao estresse podem surgir sintomas de insônia, mudança de humor, raiva, enxaquecas e dores inespecíficas ${ }^{6}$.

Nessa pandemia de COVID-19, o estresse tem sido um fator de adoecimento psicológico não só para os profis- sionais de saúde, mas para todos que tiveram suas vidas afetadas, inclusive os estudantes.

Diante disso o presente relato tem por objetivo descrever a experiência nova e única do isolamento social de acadêmicos de enfermagem no período de pandemia da COVID-19.

\section{MÉTODO}

Tipo de estudo: Estudo descritivo do tipo relato de experiência do isolamento social de acadêmicos de enfermagem de uma instituição pública de nível superior no município de Manaus/Amazonas, com foco nos fatores estressores que permeiam a interrupção das aulas, do curso e o tempo ocioso durante a pandemia de COVID-19.

Cenário de estudo: Município de Manaus/Amazonas, onde foram adotadas medidas de isolamento e suspensão das aulas, com isso adotou-se o acesso remoto home office. As discussões do relato entre os discentes participantes do relato e os docentes orientadores se deu a partir de videoconferências em plataformas virtuais que permitiram o debate crítico desta temática de acordo com as medidas de isolamento social.

Período de realização da experiência: Iniciou-se a partir da Portaria $N^{\circ}$ 626, de 13 de março de 2020 que suspendeu as atividades presenciais da instituição, em meados de março de 2020 até ao término desse relato, junho de $2020^{7}$.

Sujeitos envolvidos: Acadêmicos do terceiro e nono periodo de Enfermagem de uma instituição pública.

Aspectos éticos: Devido ao tipo de estudo não houve necessidade de submetê-lo à aprovação do Comitê de Ética em Pesquisa. Ressaltamos que os aspectos éticos foram adotados e não houve menção ou caracterização dos sujeitos para não serem identificados.

\section{OBJETIVO DA EXPERIÊNCIA}

Descrever a experiência do isolamento social de acadêmicos de enfermagem no período de pandemia de COVID-19.

\section{DESCRIÇÃO DA EXPERIÊNCIA}

A estadia compulsória em casa ocasionou diversos sentimentos, que oscilou entre descrença e esperança, associados à preocupação, ansiedade e ociosidade. O contexto não propiciou a efetividade em cumprir tarefas, por conseguinte o sentimento de "inutilidade". Ainda que houvesse conhecimento da existência de plataformas e sites gratuitos de capacitação, educação continuada, foi árduo manter a concentração e disciplina diante da si- 
tuação em que a cidade da referida instituição de ensino se encontra. Sem uma previsão para que as atividades acadêmicas retornassem, também gerou sentimento de incerteza "Quando irei me formar? Quando conseguirei um emprego? Quando cooperarei economicamente com minha familia? ". A pandemia fez com que as pessoas se colocassem em isolamento social, os acadêmicos, tinham uma jornada de estudo integral, e ao invés de passarem seus dias desenvolvendo suas atividades, passaram a ficar integralmente em casa. No início, deu-se uma falsa sensação de descanso, pois, apesar de ser um tempo de reclusão para proteção contra a contaminação do vírus, indiretamente também seria um tempo para o autocuidado, no entanto, essa sensação logo foi substituída por um estado de alerta constante, pois, apesar de todas as medidas de saúde para proteção, o receio de contaminação e da disseminação do vírus foi exacerbado, além disso, o perdurar do isolamento fez com que o nível de estresse se elevasse dificultando a concentração, isso tornou boa parte do tempo improdutivo, e acarretou em uma experiência negativa. $\mathrm{O}$ isolamento social serviu de precursor para sentimentos e sensações nocivas como irritabilidade, ansiedade, alterações no sono, falta de lazer apropriado, distanciamento dos familiares e entes queridos, dores inespecíficas, entre outros. Esses fatores estressores relatados instauraram um quadro de estresse contínuo comprometendo a saúde mental e física dos acadêmicos.

\section{PRINCIPAIS RESULTADOS ALCANÇADOS}

A pandemia, que ultrapassou as fronteiras, se propagando por todo o mundo, até o momento, gera na comunidade um sentimento de angústia e incerteza em diversas instâncias. No contexto da comunidade acadêmica da universidade federal não seria diferente, o isolamento afastou os acadêmicos de suas atividades curriculares presenciais e consequentemente gera um considerável impacto psíquico. Porém, partindo do pressuposto que a educação é concebida como um processo de transformação permanente ${ }^{8}$ devido aos acontecimentos, moldou-se à nova realidade, visto o grande crescimento de encontros acadêmicos através de lives e plataformas virtuais. Evitar a proliferação do vírus e, proteger a integridade física da população tem sido alguns dos objetivos do isolamento social. Esta medida se fez extremamente necessária, pois, por se tratar de um novo vírus, sem vacina ou comprovações científicas de remédios eficazes para a cura ou tratamento, o isolamento tornou-se mais eficaz para a diminuição da curva de contágio, especialmente para proteção dos individuos do grupo de risco, contudo, é importante entender as consequên- cias psicossociais acarretadas por essa ação. Existem evidências de surtos infecciosos anteriores, Influenza $\mathrm{H} l \mathrm{Nl}$, Síndrome Respiratória Aguda Grave (SARS), Ebola, onde constata-se que medidas de isolamento são necessárias para o controle da doença, apesar disso traz consigo experiências psicológicas negativas ${ }^{2}$. São agentes estressores que surgem durante e após o isolamento ou quarentena, como: tempo de duração do isolamento, medo de infecção, frustração e tédio, suprimentos inadequados, informações inadequadas, finanças, estigma. Apesar da nocividade que o estresse apresenta, é válido compreender que se trata de uma resposta natural do organismo frente a estímulos novos ou diferentes vividos, o corpo reage gerando respostas que refletem tanto na saúde mental como física da pessoa, até que a mesma possa adaptar-se. Dito isso é importante que haja um equilibrio e principalmente controle do estresse e seus componentes, a fim de evitar sua demasia?. Quando os fatores estressores não são previstos e não são atenuados, os indivíduos podem sofrer consequências mais danosas em longo prazo, como transtorno de estresse pós-traumático e depressão ${ }^{10}$. Eventos traumáticos dos surtos de pandemias atrelados aos fatores estressantes do isolamento social ou quarentena, podem levar o indivíduo a desenvolver depressão, pois, quando não tratados inicialmente os sintomas do estresse podem permanecer gerando outros sentimentos prejudiciais, como, apatia, desmotivação, medo, culminando em um quadro depressivo $^{11}$. Apesar de o isolamento social causar consequências psicológicas negativas, não o adotar pode ocasionar em danos ainda piores, apesar disso, é importante e necessário adotar medidas para mitigar seus efeitos adversos ${ }^{2-11}$. O estado do Amazonas foi um dos estados mais acometidos e por conseguinte Manaus, a capital do estado, foi a cidade mais atingida do estado. Demonstrou letalidade de 5,63\% no mês de maio em todo o estado, tendo atingido a taxa de 8,09\%, no mês de abril ${ }^{3-4}$.

Esse tipo de informação que foi constantemente difundida nos meios midiáticos, e muitas vezes acompanhadas de notícias inverídicas, causou amedrontamento intensificado na população como um todo e principalmente em acadêmicos da área da saúde, pois sabiam que viriam a enfrentar essa responsabilidade, direta ou indiretamente. Embora a pandemia tenha estimulado pensamentos pessimistas, hábitos ruins e um sentimento de desesperança, também suscitou uma consciência na comunidade em geral sobre a valorização da categoria profissional da Enfermagem. Um exemplo disso foi o Projeto de Lei n 2564 de 2020 que institui um novo piso salarial nacional do Enfermeiro, Técnico de Enfermagem, do Auxiliar de Enfermagem e 
da Parteira ${ }^{12}$. Essas medidas advindas dos representantes políticos ressignificam o valor do profissional perante a sociedade, não somente no meio acadêmico científico.

\section{Limitações da experiência}

Como limitador do estudo, reiteramos que as percepções deste relato são compostas de uma pequena parcela de acadêmicos, sendo necessário outros estudos mais abrangentes desta temática na região.

\section{Contribuições para a prática}

Esse relato se faz necessário diante do atual cenário, único e nunca antes vivenciado, dada as proporções mundiais que o vírus alcançou. Sendo de importância o isolamento social para impedir a proliferação, ainda maior, do vírus e proteção aos indivíduos e apesar dos benefícios já citados, é importante evidenciar os danos que o mesmo proporciona. Ao refletir sobre as consequências e limitações ocasionadas pela pandemia, podemos repensar nas práticas e soluções para o cotidiano, assim como o planejamento de políticas públicas que venham contribuir com o aperfeiçoamento profissional, apoio institucional e psicológico. Além do aprimoramento de habilidades psíquicas e sociais para contribuir com a população através de informações fundamentadas cientificamente sobre a COVID-19, seja por meio das redes sociais ou pela procura espontânea, justamente pelo reconhecimento da Enfermagem em meio à pandemia. Portanto, é relevante colocá-los em evidência para que medidas de autocuidado sejam tomadas para que não sofram efeitos danosos em longo prazo.

\section{CONSIDERAÇÕES FINAIS}

É unânime afirmar que a pandemia foi um imprevisto na sociedade em geral. Causou sobressaltos de diversas intensidades, porém quem estava inserido no âmbito da saúde vivenciou uma comoção demasiada. As sensações mais diversificadas afligiram esse grupo, principalmente porque essa área está diretamente ligada a pesquisas científicas e assistência para os que necessitam.

Para a comunidade acadêmica, além dos sentimentos prejudiciais, sucedeu a expectativa de um futuro melhor, com pensamentos empáticos para com a sociedade e convicção que a Enfermagem será mais valorizada, bem como os profissionais que atuam nela. O tempo sem aulas teórico-práticas pode ser usufruído para produção de trabalhos e retomada de projetos que haviam sido postergados durante o período acadêmico normal, visto que a prioridade do período habitual seria priorizada o preparo para exames avaliativos e demais atividades.

É primordial que, a partir do atual momento, haja um acompanhamento psicossocial para os que enfrentam esse dilema, pois não é algo trivial, a geração nunca encarou uma situação como esta.

No entanto é necessário adquirir resiliência e inteligência emocional, visto que a escolha pela graduação em Enfermagem traz consigo esse ônus.

Contribuições dos Autores: Concepção do estudo: SVC, DSG e NFCCR; Coleta análise e interpretação dos dados: DSG, NFCCR, TNR; Redação e revisão crítica do manuscrito: DDSG, NFCCR. TNR, SVC, AAF; Aprovação da versão final a ser publicada: AAF, SVC.

Agradecimentos:

À Universidade Federal do Amazonas.

\section{REFERÊNCIAS}

1. Ministério da Saúde (BR). O que é COVID-19. [Internet] [cited 2020 jul 05]. Available from: https://coronavirus.saude.gov.br/sobre-a-doenca\#o-que-e-covid

2. Brooks SK, Webster RK, Smith LE, Woodland L, Wessely PS, Greenberg PN, et al. The psychological impact of quarantine and how to reduce it: rapid review of the evidence. Rev The Lancet [Internet], 2020 [cited 2020 may 29], 395 (10227): 912-920 Available from: https:// www.thelancet.com/journals/lancet/article/PIIS0140-6736(20)30460-8/fulltext.

3. Fundação de Vigilância em Saúde do Amazonas. Boletins Diários de Casos de COVID-19 [Internet]. 28 Apr 2020 [cited 2020 may 29]. Available from: http://www.fvs.am.gov.br/media/publicacao/BOLETIM_DI\%C3\%81RIO_DE_CASOS_COVID-19_28-04-2020.pdf 
4. Fundação de Vigilância em Saúde do Amazonas. Boletins Diários de Casos de COVID-19 [Internet] 28 May 2020 [cited 2020 may 29]. Available from: http://www.fvs.am.gov.br/media/publicacao/BOLETIM_DI\%C3\%81RIO_DE_CASOS_COVID-19_28-05-2020.pdf

5. Parvan K, Zamanzadeh V, Dizaji SL, Mousavi SM, Safaie N. Patient's Perception of Stressors Associated with Coronary Artery Bypass Surgery. J Cardiovasc Thorac Res [Internet] 5(3):113-7. 2013 [cited 2020 may 28]. Available from: https://jcvtr.tbzmed.ac.ir/Article/JCVTR_549_20140621075336

6. Ko YJ, Jung MS, Park KS. Effects of Aroma Inhalation Method on Test Anxiety, Stress Response and Serum Cortisol in Nursing Students. J Korean Acad Fundam Nurs, [Internet] 20(4). 2013 [cited 2020 may 28] Available from: http://www.koreascience.or.kr/article/ JAKO201336161063505.page

7. Ministério da Educação (BR). Universidade Federal do Amazonas. Gabinete do Reitor. Suspende as atividades presenciais acadêmicas e administrativas. Portaria № 626, de 13 de Março de 2020. [Internet] 2020 [cited 2020 june 10]. Available from: https://edoc.ufam. edu.br/bitstream/123456789/3061/1/SEI_23105.010531_2020_90.pdf

8. Fernades HIVM, Rolim KMC, Figueiredo MCB. O significado do cuidar humanizado: vivências ensino-aprendizagem dos alunos do curso de licenciatura. Rev Enferm. Foco [Internet], 2011 [cited 2020 june 08]. Available from: http://revista.cofen.gov.br/index.php/enfermagem/article/view/192

9. Brito MDC, Araújo RMB, Dias MSDA, Silva LCC, Rodrigues PDV, Moita MP. Análise da ocorrência de estresse entre estudantes de enfermagem. Rev Enferm. Foco [Internet], 2019 [cited 2020 june 08]. Available from: http://revista.cofen.gov.br/index.php/enfermagem/ article/view/2391/653

10. Hawryluc L, Gold WL, Robinson S, Pogorski S, Galea S, Styra R. SARS Control and Psychological Effects of Quarantine, Toronto, Canada. Rev Emerging Infectious Diseases, [Internet] 10(7): 1206-1212 2004 [cited 2020 may 29]. Available from: https://www.ncbi.nlm.nih. gov/pmc/articles/PMC3323345/.

11. Liu X, Kakade M, Fuller CJ, Fan B, Fang Y, Kong J, Guan Z, Wu P. Depression after exposure to stressful events: lessons learned from the severe acute respiratory syndrome epidemic. Rev Comprehensive Psychiatry [Internet] 53(1): 15-23, 2012 [cited 2020 may 29]. Available from: https://pubmed.ncbi.nlm.nih.gov/21489421/

12. Brasil. Projeto de Lei $n^{\circ} 2564$ de 2020 (BR) [Internet]. Altera a Lei no 7.498, de 25 de junho de 1986, para instituir o piso salarial nacional do Enfermeiro, do Técnico de Enfermagem, do Auxiliar de Enfermagem e da Parteira. 12 may 2020 [cited 2020 may 29]. Available from: https://www25.senado.leg.br/web/atividade/materias/-/materia/141900 